\title{
Unveiling the Stable Nature of LiPON-associated Electrode/Electrolyte Interphases via Cryogenic Electron Microscopy
}

Diyi Cheng $^{1}$, Ryosuke Shimizu ${ }^{1}$, Jamie Weaver ${ }^{2}$ and Y. Shirley Meng ${ }^{3}$

${ }^{1}$ University of California, San Diego, United States, ${ }^{2}$ NIST, Material Measurement Laboratory, United

States, ${ }^{3}$ University of California San Diego, La Jolla, California, United States

The past four decades have witnessed intensive research efforts on the chemistry, structure, and morphology of the solid electrolyte interphase (SEI) in Li-metal and Li-ion batteries (LIBs) using liquid or polymer electrolytes, since the SEI is considered to predominantly influence the performance, safety and cycle life of batteries. ${ }^{1-3}$ Pioneering work by Peledet $a l .{ }^{4}$ and Aurbachet al. ${ }^{5}$ has independently proposed two widely accepted SEI models - a mosaic SEI and a multilayer SEI - to explain the structural and chemical evolution mechanism during the SEI formation. Although the studies on SEI chemistry and morphology formed by using various electrolyte compositions and electrode materials have been well documented in literature, existing models still require further efforts to be truly validated in terms of the distribution of nanostructures within the SEI layer. The dearth of SEI studies for solid-state electrolytes (SSE) also leaves the SEI formation mechanism at the Li/SSE interphase elusive.

Compared with their liquid-electrolyte analogues, SSEs have drawn increased attention as they promote battery safety, exhibit a wide operational temperature window, and improve energy density by enabling Li metal as anode materials for next-generation lithium-ion batteries. Despite suitable mechanical properties to prevent $\mathrm{Li}$ dendrite penetration, relatively wide electrochemical stability windows, comparable ionic conductivities, and intrinsic safety, most SSEs are found to be thermodynamically unstable against Li metal, where SSE decomposition produces a complex interphase, analogous to the SEI formed in liquid electrolyte systems. An ideal passivation layer should consist of ionically conductive but electronically insulating components to prevent the SSE from being further reduced.

As one of the most successful SSEs, LiPON has enabled an all-solid-state thin-film battery with a Li metal anode and a high-voltage $\mathrm{LiNi}_{0.5} \mathrm{Mn}_{1.5} \mathrm{O}_{4}$ (LNMO) cathode to achieve a capacity retention of $90 \%$ over 10,000 cycles with a Coulombic efficiency over $99.98 \%,{ }^{6}$ indicating the presence of extremely stable interphases between LiPON and electrode materials. The superior electrochemical performance of LiPON against Li metal has attracted numerous research efforts aiming to understand the underlying nature of stable Li/LiPON interphase. Experimental efforts to identify this stable interphase of LiPON against Li metal, however, have been impeded by the limited characterization techniques available due to the low interaction volume of lithium, the amorphous nature of LiPON, and the extreme susceptibility of both lithium metal and LiPON to ambient air and probe damage. ${ }^{7,8}$

Originating from the structural biology field, cryogenic focused ion beam (cryo-FIB) and cryogenic electron microscopy (cryo-EM) have recently been introduced to battery research, and have proven the ability to preserve and probe Li metal for quantitative structural and chemical analysis. ${ }^{8-10}$ Given the susceptibility of LiPON and Li under electron beam exposure, ${ }^{11}$ herein we combined cryo-FIB and cryoEM to preserve the Li/LiPON interphase and characterize its chemistry and structure. ${ }^{12}$ A cryo-liftout method is applied for transferring a lamella from the bulk sample to the FIB grid at low temperature to avoid the contamination from organometallic Pt or amorphous ice. (Fig. 1A\&B). By preserving and probing Li/LiPON interphase in cryo-EM, we observed concentration gradients of nitrogen and phosphorous into Li metal (Fig. 1C-F), and a $<80 \mathrm{~nm}$ thick interphase consisting of a distribution of 
crystalline decomposition products embedded within an amorphous matrix. (Fig. 2A) The observed structural and chemical evolution across the interphase identifies the SEI components to be $\mathrm{Li}_{2} \mathrm{O}, \mathrm{Li}_{3} \mathrm{~N}$ and $\mathrm{Li}_{3} \mathrm{PO}_{4}$, with a unique multilayer-mosaic distribution (Fig. 2B-I), confirmed by XPS depth profiling. According to these findings, we proposed a multilayer-mosaic SEI model. (Fig. 2J) and further proposed a reaction mechanism for $\mathrm{Li} / \mathrm{LiPON}$ interphase, stressing the diffusion of decomposition product species and structural reconfiguration during equilibration. A combination of neutron depth profiling (NDP) and cyro-EM for elucidating the interfacial chemistry and structure between LNMO cathode and LiPON will also be covered, which offers valuable insights on LiPON's stability against high voltage cathode.
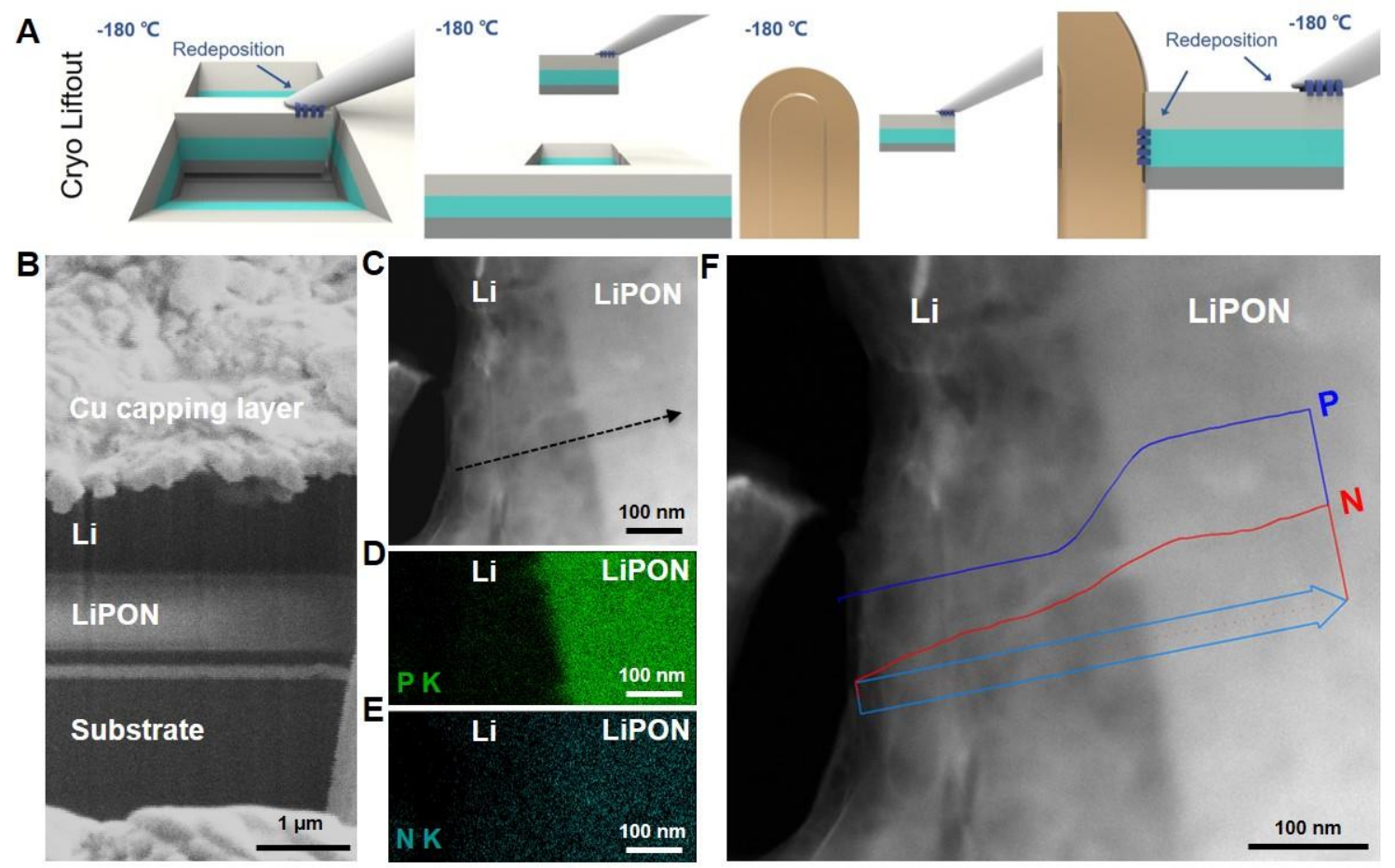

Figure 1. Cryo-liftout methodology and cryo-STEM EDS results. (A) Schematic for the cryo-liftout methodology. (B) Cryo-FIB-SEM cross-sectional image of the Li/LiPON sample. (D) Cryo-STEM DF image of Li/LiPON interface. EDS mapping results of $\mathrm{P}(\mathrm{E})$ and $\mathrm{N}(\mathrm{F})$ signals in the region shown in (D). (G) EDS line-scan of $\mathrm{P}$ and $\mathrm{N}$ signals (counts per second) along the black dashed arrow in (D). 

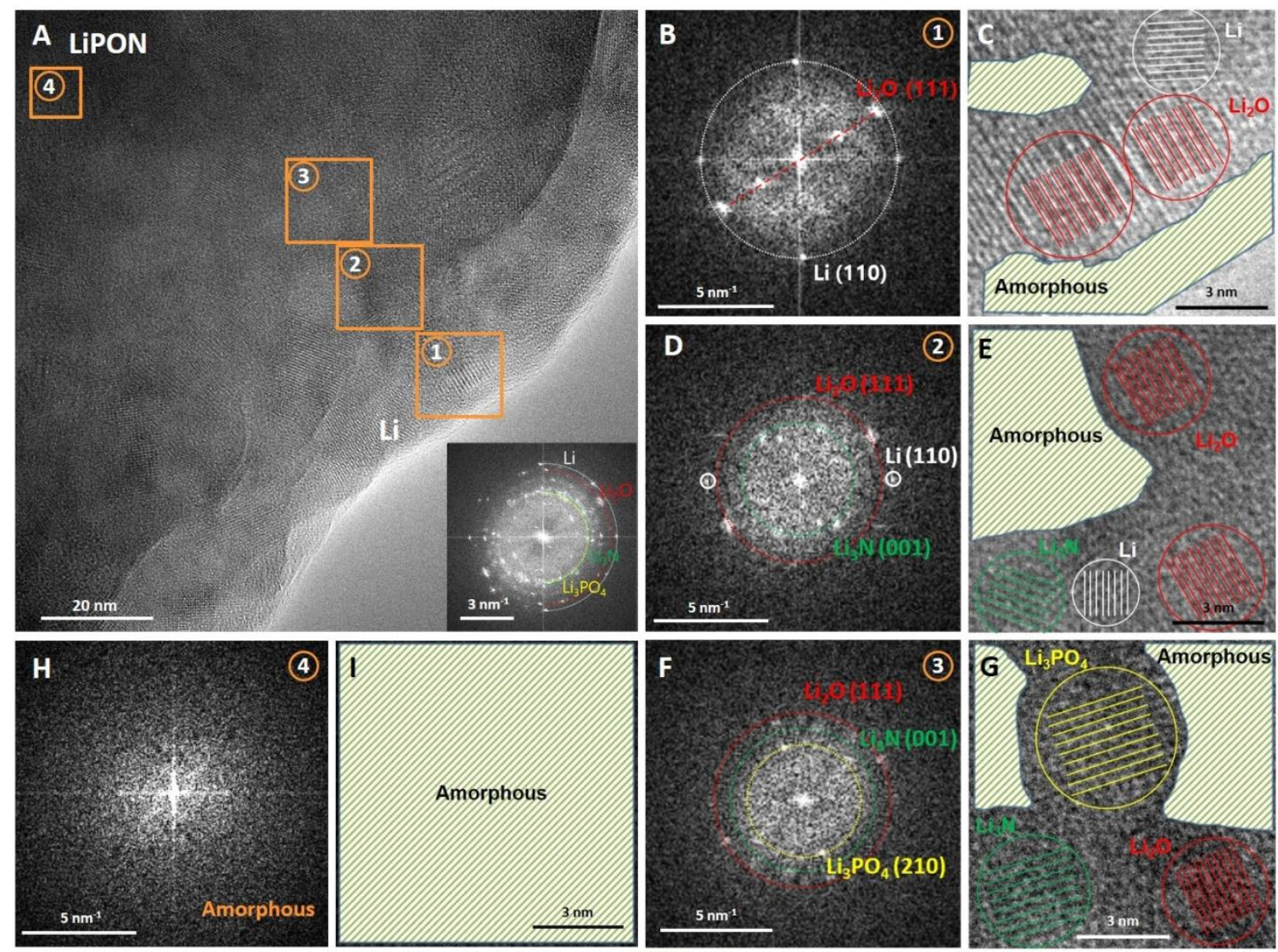

J

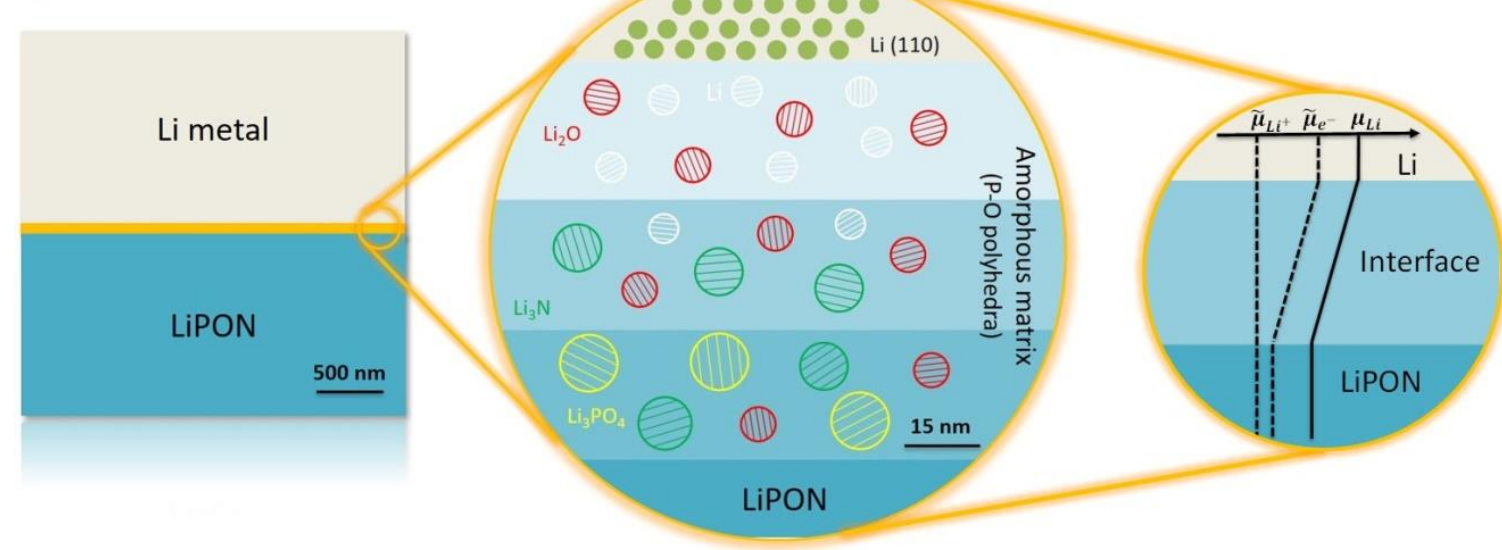

Figure 2. Nanostructures of Li/LiPON interphase and proposed interphase model. (A) HRTEM image of the interphase where four regions (region 1-4) are highlighted by orange squares to indicate different stages of the multilayered structure across the interphase. Inset image is the FFT result of the whole area in (A). (B, D, F \& H) FFT patterns corresponding to region 1-4. (C, E, G \& I) Nanostructure schematic corresponding to region 1-4. (J) Interphase model proposed from this work.

\section{References}


1. Aurbach, D. Review of selected electrode-solution interactions which determine the performance of Li and Li ion batteries. J. Power Sources 89 (2000), 206-218.

2. Se, T. H. E., Electrolyte, M. \& Batteries, T. O. L. The SEI model-application electrolyte. Elecrrochimica Acta. 40 (1995), 2197-2204.

3. Wang, A., Kadam, S., Li, H., Shi, S. \& Qi, Y. Review on modeling of the anode solid electrolyte interphase (SEI) for lithium-ion batteries. npjComput. Mater. 4 (2018), 15.

4. Peled, E. The Electrochemical Behavior of Alkali and Alkaline Earth Metals in Nonaqueous Battery Systems-The Solid Electrolyte Interphase Model. J. Electrochem. Soc. 126 (1979), 2047.

5. Aurbach, D. The Surface Chemistry of Lithium Electrodes in Alkyl Carbonate Solutions. J. Electrochem. Soc. 141 (1994), 1.

6. Li, J., Ma, C., Chi, M., Liang, C. \&Dudney, N. J. Solid electrolyte: The key for high-voltage lithium batteries. Adv. Energy Mater. 5 (2015), 1-6.

7. Yu, X. A Stable Thin-Film Lithium Electrolyte: Lithium Phosphorus Oxynitride. J. Electrochem. Soc. 144 (1997), 524.

8. Wang, X., Zhang, M., Alvarado, J., Wang, S., Sina, M., Lu, B., Bouwer, J., Xu, W., Xiao, J., Zhang, J. G., et al. New Insights on the Structure of Electrochemically Deposited Lithium Metal and Its Solid Electrolyte Interphases via Cryogenic TEM. Nano Lett. 17 (2017), 7606-7612.

9. Singh, C. N., Butler, K. T., MacDonald, A. H., Piper, L. F. J. \& Lee, W.-C. Dynamic disorder induced memristance in amorphous solids. (2019) arXiv:1908.08070.

10. Li, Y., Li, Y., Pei, A., Yan, K., Sun, Y., Wu, C. L., Joubert, L. M., Chin, R., Koh, A. L., Yu, Y., et al. Atomic structure of sensitive battery materials and interfaces revealed by cryo-electron microscopy. Science 358 (2017), 506-510.

11. Santos-Ortiz, R., Rojhirunsakool, T., Jha, J. K., Al Khateeb, S., Banerjee, R., Jones, K. S. \& Shepherd, N. D. Analysis of the structural evolution of the SEI layer in FeF2 thin-film lithium-ion batteries upon cycling using HRTEM and EELS. Solid State Ionics 303 (2017), 103-112.

12. Cheng, D., Wynn, T. A., Wang, X., Wang, S., Zhang, M., Shimizu, R., Bai, S., Nguyen, H., Fang, C., Kim, M., et al. Unveiling the Stable Nature of the Solid Electrolyte Interphase between Lithium Metal and Lipon Via Cryogenic Electron Microscopy. Joule, 4 (2020), 11, 2484-2500 